\title{
Direct retrieval of Kerr and plasma effects from alignment-induced spatiotemporal modulation
}

\author{
Yahui Feng, ${ }^{1}$ Wenxue Li, ${ }^{1,2}$ Jia Liu, ${ }^{1}$ Haifeng Pan, ${ }^{1}$ Jian Wu, ${ }^{1}$ and Heping Zeng,* \\ ${ }^{1}$ State Key Laboratory of Precision Spectroscopy, East China Normal University, Shanghai 200062, China \\ ${ }^{2}$ e-mail: wxli@phy.ecnu.edu.cn \\ *Corresponding author: hpzeng@phy.ecnu.edu.cn
}

Received June 12, 2012; accepted July 11, 2012;

posted July 20, 2012 (Doc. ID 170442); published September 12, 2012

\begin{abstract}
We developed a direct measurement method for comprehensive analyses of Kerr and plasma effects that cooperated or competed with molecular alignment-induced spatiotemporal modulation for intense aligning and weak probing pulses around zero time delay. The mixed influences were revealed by time-resolving the combined spatial focusing or defocusing dynamics under different molecular alignment directions and degrees. The nonlinear refractive index and plasma density were extracted straightforwardly, facilitating accurate explorations on multiphoton ionization and nonlinear optical Kerr effects of aligned molecular gases. (c) 2012 Optical Society of America

OCIS codes: $320.7110,020.2649,190.7110$.
\end{abstract}

Molecular alignment [1] through impulsive rotational Raman excitation has inspired vigorous explorations in the microscopic domain to reveal molecular dynamics and structures for molecular physics and chemistry, such as, full-dimensional molecular manipulation [2], high-order harmonic generation [3], molecular-orbital reconstruction [4], and revivable ultrafast optical buffer with molecular rotational wave-packets []ㅡ, as well as in the macroscopic domain to control optical properties of the molecular gaseous media for intriguing alignmentbased applications, such as alignment-induced spatiotemporal modulation []], ultrashort pulse compression []], filamentary intense laser pulse propagation [ㅁ-10], and enhanced broadband supercontinuum generation $[\underline{8}, 11]$. Alignment-induced spatiotemporal modulation could facilitate a convenient cross-defocusing measurement to distinguish parallel, perpendicular, and random alignment according to the alignment-induced spatial gradient of the refractive index. Besides the alignmentmediated interaction of the ultrashort laser pulse, the intensity-dependent Kerr effect and orientation-sensitive plasma defocusing also play important roles in the modulating of the refraction index at the zero time delay. All those effects may cooperate or compete with mixed contributions in altering nonlinear interaction of aligning and probing pulses, nonlinear propagation, and alignment-based optical nonlinearities [8]. Accordingly, it is actually inaccurate to analyze the molecular alignment effect without taking the nonnegligible Kerr and plasma contributions into account. A handy method is thus desired to distinguish individual impacts and reflect the cooperative or competitive interferences of relevant effects, including alignment-based spatiotemporal or spectral modulation, Kerr optical nonlinearities, alignment-dependent multiphoton ionization, and plasma defocusing.

In this Letter, we developed a direct measurement method to distinguish cooperation and competition of Kerr and plasma effects with alignment-induced spatiotemporal modulation for synchronized intense aligning and weak probing pulses. The combined spatial focusing or defocusing dynamics of collinearly propagated probe pulse were time-resolved to reveal individual influences of the relevant nonlinear effects under different molecular alignment directions and degrees. The nonlinear refractive index and multiphoton ionization-induced plasma density were extracted straightforwardly, facilitating accurate explorations on the high-order nonlinear Kerr optical effect $[\underline{8}, \underline{12}]$ and orientation-sensitive nonlinear optical responses of aligned molecules. It may also provide accurate information to get a clear understanding of nonlinear interaction of intense ultrashort laser pulses in aligned molecule gases.

Our experiment was carried out with the output from an amplified Ti:sapphire laser system (60 fs, $800 \mathrm{~nm}$, $1 \mathrm{kHz}$ ), which was split into two parts by a beam splitter. The reflected beam went through a beta barium borate (BBO) crystal (type-I, $29.2^{\circ}$-cut) to generate the probe pulse $\left(400 \mathrm{~nm}, 2.85 \times 10^{12} \mathrm{~W} / \mathrm{cm}^{2}\right)$, while the transmitted one acted as the pump pulse with the pulse energy adjustable. A half-wave plate located in the pump arm was employed to change the pulse polarization. The two pulses were focused collinearly into a gas-filled cell by using lenses of $f=40$ and $50 \mathrm{~cm}$ with their focal spots spatially overlapped. After the gas cell, the probe pulse was imaged by a $4-f$ system and only the central part was chosen to be focused onto a photodiode detector [13]. A lock-in amplifier was used to further improve the signal-to-noise ratio. Since the photodiode actually integrated the selected part of the probe pulse, the measured probe intensity variation, which reflected spatial focusing or defocusing, was proportional to the refractive index change in the collinear interaction region. After a long collinear interaction $(\sim 20 \mathrm{~cm})$, the induced focusing/defocusing could bring about observable variation of the probe beam profile. A signal peak and valley could be reached for the pump-induced focusing and defocusing, respectively.

Molecules excited by a spatially Gaussian-shaped pump pulse functioned equivalently as a positive (or negative) lens for the probe pulse polarized parallel (or perpendicularly) along the aligned molecular axis [14], resulting in an observable increase (or decrease) of the measured probe intensity that revealed Gaussian gradient alignment-induced variation of the refractive index, 


$$
\begin{gathered}
\Delta n_{\|}=\frac{2 \pi \rho_{0} \Delta \alpha}{n_{0}}\left[\left\langle\cos ^{2} \theta_{\|}\right\rangle-1 / 3\right], \\
\Delta n_{\perp}=-\frac{\pi \rho_{0} \Delta \alpha}{n_{0}}\left[\left\langle\cos ^{2} \theta_{\perp}\right\rangle-1 / 3\right],
\end{gathered}
$$

where $\rho_{0}$ denotes the initial molecular density, $\Delta \alpha$ is the molecular polarizability anisotropy, $\Delta \alpha=\alpha_{\|}-\alpha_{\perp}$, and $\alpha_{\|}$and $\alpha_{\perp}$ are the polarizability components parallel and perpendicular to the molecular axis.

For the pump intensity used in our experiments, phase modulation between the pump and probe pulses induced observable intensity-dependent Kerr focusing. As the synchronized pump and probe pulses were polarized parallel or perpendicularly, the nonlinear refractive index variation was given by

$$
\begin{aligned}
& \Delta n_{\|}=2 n_{2} I_{\text {pump }}, \\
& \Delta n_{\perp}=\frac{2}{3} n_{2} I_{\text {pump }},
\end{aligned}
$$

where $n_{2}$ is the nonlinear refractive index, and $I_{\text {pump }}$ is the excited pulse intensity.

Plasma defocusing decreased the detected intensity, due to plasma-induced refractive index change, expressed as $\Delta n=-2 \pi e^{2} N_{e} /\left(m_{e} \omega_{0}^{2}\right)$, where $\omega_{0}, e, N_{e}$, and $m_{e}$ denote the angular frequency of the probe pulse, electron charge, plasma density, and electron mass.

Figure 1 presents the qualitative features of independent Kerr, plasma, and molecular alignment contributions around zero time delay. The time zero was set at the emerging time of the peak of Kerr effect, which was also the moment that the Gaussian-shaped pump

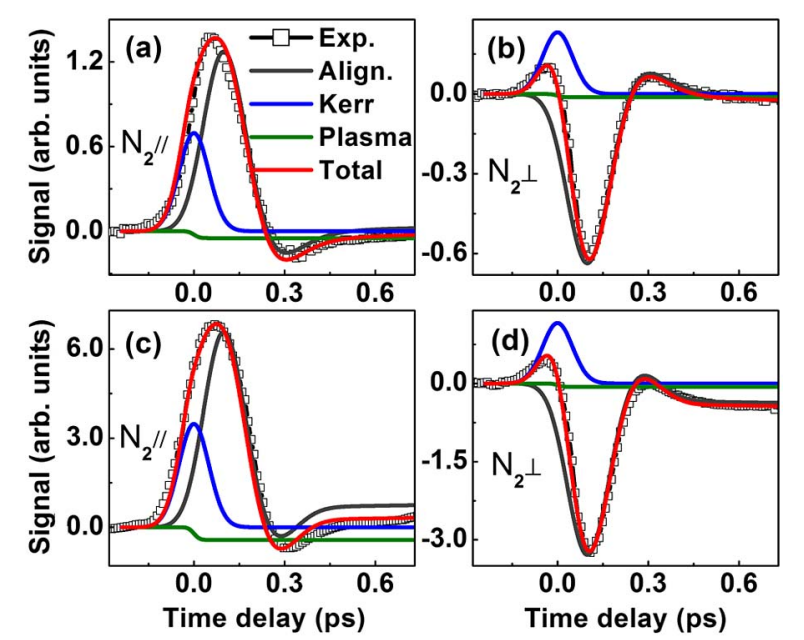

Fig. 1. (Color online) Experimental signal (open squares), simulation of the molecular alignment (gray line); Kerr (blue line), plasma (olive line), and total contributions (red line) of $1 \mathrm{~atm} . \mathrm{N}_{2}$ at room temperature around zero time delay. The probe pulse was polarized parallel (a), (c) and perpendicularly (b), (d), represented by $\mathrm{N}_{2 \|}$ and $\mathrm{N}_{2 \perp}$, with respect to the prealigned $\mathrm{N}_{2}$ molecules under the pump pulses of (a), (b) 0.37 and (c), (d) $1.84 \times 10^{13} \mathrm{~W} / \mathrm{cm}^{2}$. pulse reached its maximum due to the instantaneous impulsive response. The strongest signal of the molecular alignment occurred about 100 fs later owing to the inertial delay. The multiphoton ionization-induced plasma defocusing started with the impulse of the pump pulse, reached its maximum after the pump, and then remained almost a constant for a temporal range of several to tens of picoseconds. By comparing Fig. 1(a) with Fig. 1(b) and Fig. 1(c) with Fig. 1(d), we could infer that Kerr focusing signals for the parallel and perpendicularly polarized pump and probe pulses exhibited an amplitude ratio close to 3, exactly following the anticipation from Eqs. (3) and (4), while the molecular alignment induced a measured signal change ratio close to 2 , coinciding with the relevance $\left\langle\cos ^{2} \theta_{\|}\right\rangle+2\left\langle\left\langle\cos ^{2} \theta_{\perp}\right\rangle=1\right.$. Rather than the linear increase depending on the pump intensity and polarization that both Kerr and molecular alignment effects obeyed, the plasma contribution exhibited an exponential growth, which could be clearly obtained by the comparisons of Fig. 1(a) with Fig. 1(c) and Fig. 1(b) with Fig. 1(d). As the molecules were aligned parallel with the probe field polarization [Figs. 1(a) and 1(c)], the Kerr focusing cooperated with the molecular alignmentinduced focusing, pulling the combined signal peak to shift approximately 30 fs forward with respect to the molecular alignment peak. On the contrary, for the perpendicular case, the Kerr focusing counteracted molecular alignment-induced defocusing, a small side peak turned out nearly 30 fs ahead of the Kerr peak, and the total signal valley was pushed about 10 fs away.

Figure 2 shows the signal change with the increase of the pump intensity. Figures 2(a) and 2(b) display the simulated molecular alignment at its first revival, which moves slightly forward as the pump pulse intensity increases. Higher pump intensity induced a stronger torque, which gave a little earlier and more powerful kick to molecules, and thus decreased the inertial delay. As observed in the experiments, the Kerr effect, molecular alignment, and plasma impacts acted jointly to have the total signal peak [Fig. 2(c)] and valley [Fig. $\underline{2(\mathrm{~d})}$ ] pushed

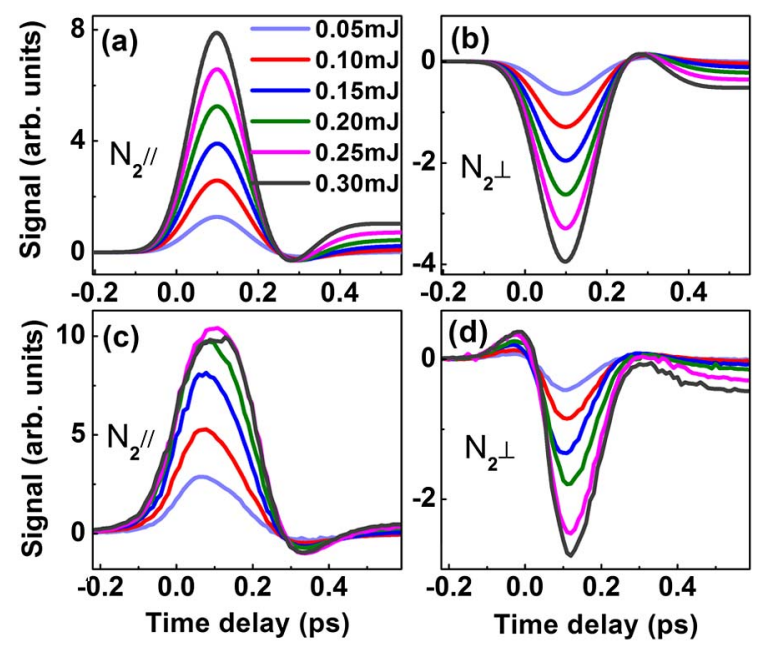

Fig. 2. (Color online) Simulated (a), (b) and measured (c), (d) molecular alignment signals at its first revival as the probe was polarized parallel (a), (c) and perpendicularly (b), (d) to the pump with pump energy varied. 


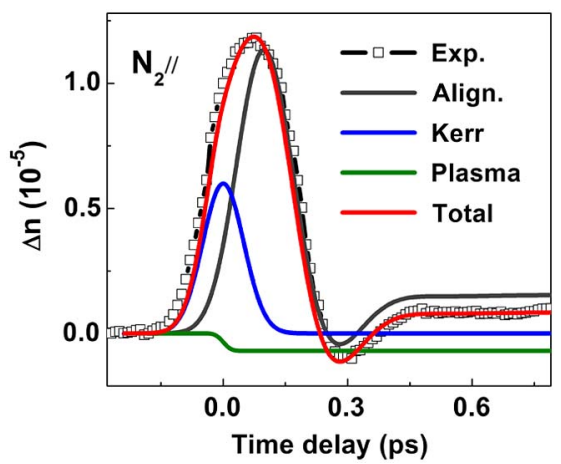

Fig. 3. (Color online) Corresponding refractive index variation caused by the molecular alignment (gray line), Kerr (blue line), and plasma (olive line) when the probe pulse was polarized parallel with the pump $\left(\sim 22.1 \mathrm{TW} / \mathrm{cm}^{2}\right)$. The experimentally measured and total refractive index changes are depicted as squares and a red line.

backward with the increase of the pump energy. As the probe was parallel polarized with the prealigned molecules, the Kerr effect cooperated with alignment, while plasma produced a contrast impact. Due to different response time, plasma defocusing set more influence on molecular alignment than the Kerr effect. However, in the perpendicular situation, the Kerr effect counteracted alignment contribution, so the sum of the alignment and plasma made the signal valley move backward.

As shown in Fig. 1, our simulation of the combined contributions from Kerr, plasma, and molecular alignment agrees well with the experimental results, indicating that our method to reconstruct all the three effects is valid in both time and intensity scales. Figure 3 provides the calculated refractive index changes induced by Kerr, molecular alignment, and plasma impacts, which were normalized on the basis of Eq. (1) under the pump intensity of $22.1 \mathrm{TW} / \mathrm{cm}^{2}$. In the simulation, $\Delta \alpha=0.93 \times 10^{-24} \mathrm{~cm}^{3}, \rho_{0}=2.46 \times 10^{19} \mathrm{~cm}^{-3}$ were adopted. Once the quantitative refractive index variation was obtained, according to Eq. (3), the nonlinear coefficient $n_{2}$ could be derived as $0.136 \mathrm{~cm}^{2} / \mathrm{EW}$, which approximated to the recently reported value [15]. In addition, we could perform direct measurements on the plasma density induced by multiphoton ionization. Here, on the basis of the relationship of plasma density and plasma-induced refractive index variation, the stabilized plasma density was extracted as $1.17 \times 10^{15} \mathrm{~cm}^{-3}$ under the pump excitation pulse of $22.1 \mathrm{TW} / \mathrm{cm}^{2}$. Moreover, our results indicated that the plasma density increased sharply to $1.72 \times 10^{16} \mathrm{~cm}^{-3}$ when the pump intensity reached $33.1 \mathrm{TW} / \mathrm{cm}^{2}$, indicating that there should be a critical intensity above which the molecules were greatly ionized.
In summary, we demonstrated a straightforward method to distinguish Kerr and plasma effects from alignment-induced spatiotemporal modulation, reconstruct their collaborating or competing processes by time-resolving the combined spatial (de)focusing signal peaks or valleys of ultrashort probe pulse under different aligning pulse intensities, and retrieve the nonlinear refractive index variation caused by individual effects for synchronized aligning and probing pulses. The developed method facilitated comprehensive analyses on the relevant nonlinear optical effects of aligned molecules. It may also provide accurate information for in-depth studies on the controversial high-order nonlinear optical Kerr effects, spatiotemporal and spectral or polarization modulation, and multifilamentation.

This work was partly funded by the National Natural Science Fund (10990101 and 11004061), the National Key Project for Basic Research (2011CB808105), and the International Science and Technology Cooperation Program of China (2010DFA04410).

\section{References}

1. H. Stapelfeldt and T. Seideman, Rev. Mod. Phys. 75, 543 (2003).

2. J. J. Larsen, K. Hald, N. Bjerre, H. Stapelfeldt, and T. Seideman, Phys. Rev. Lett. 85, 2470 (2000).

3. T. Kanai, S. Minemoto, and H. Sakai, Nature 435, 470 (2005).

4. J. Itatani, J. Levesque, D. Zeidler, H. Niikura, H. Pépin, J. Kieffer, P. B. Corkum, and D. Villeneuve, Nature 432, 867 (2004).

5. J. Wu, P. Lu, J. Liu, H. Li, H. Pan, and H. Zeng, Appl. Phys. Lett. 97, 161106 (2010).

6. R. A. Bartels, T. C. Weinacht, N. Wagner, M. Baertschy, C. H. Greene, M. M. Murnane, and H. C. Kapteyn, Phys. Rev. Lett. 88, 013903 (2001).

7. J. Wu, H. Cai, H. Zeng, and A. Couairon, Opt. Lett. 33, 2593 (2008).

8. S. L. Chin, T. J. Wang, C. Marceau, J. Wu, J. Liu, O. Kosareva, N. Panov, Y. Chen, J. F. Daigle, S. Yuan, A. Azarm, W. Liu, T. Seideman, H. Zeng, M. Richardson, R. Li, and Z. Xu, Laser Phys. 22, 1 (2012).

9. F. Calegari, C. Vozzi, S. Gasilov, E. Benedetti, G. Sansone, M. Nisoli, S. De Silvestri, and S. Stagira, Phys. Rev. Lett. 100, 123006 (2008).

10. S. Varma, Y. H. Chen, and H. M. Milchberg, Phys. Rev. Lett. 101, 205001 (2008).

11. J. Wu, H. Cai, Y. Peng, and H. Zeng, Phys. Rev. A 79, 041404 (2009).

12. V. Loriot, E. Hertz, O. Faucher, and B. Lavorel, Opt. Express 17, 13429 (2009).

13. Y. Feng, H. Pan, J. Liu, C. Chen, J. Wu, and H. Zeng, Opt. Express 19, 2852 (2011).

14. J. Wu, H. Cai, Y. Peng, Y. Tong, A. Couairon, and H. Zeng, Laser Phys. 19, 1759 (2009).

15. V. Loriot, E. Hertz, O. Faucher, and B. Lavorel, Opt. Express 18, 3011 (2010). 